\title{
Template generation for continuous transfer functions using interval analysis ${ }^{\text {th }}$
}

\author{
P.S.V. Nataraj*, Gautam Sardar \\ Systems \& Control Engineering Group, Department of Electrical Engineering, Indian Institute of Technology, Bombay - 400 076, India
}

\begin{abstract}
This paper presents a template generation algorithm for transfer functions that are continuous in the uncertain system parameters. The algorithm is developed using interval mathematics. The generated templates are of arbitrary accuracy, safe, and reliable. Further, if the magnitude and phase are continuously differentiable functions of the parameters, then the algorithm can be speeded up considerably with the help of an additional step based on the interval Gauss-Seidel method. The convergence, finite termination, safety, and reliability properties of the algorithm are proven, and an example is provided to demonstrate its versatility.
\end{abstract}

Keywords: Frequency responses; Image sets; QFT; Templates; Transfer functions; Uncertain linear systems; Value sets

\section{Introduction}

A plant template is a set of complex numbers representing the frequency response at a fixed frequency of an uncertain plant transfer function. Plant templates are used in several approaches to analysis and design of robust control systems, for example, Bhattacharyya, Chapellat and Keel (1995) and QFT of Horowitz (1991). Templates are also referred to as value sets or image sets in the literature, and the problem of computing the template at a given frequency is known as the template generation problem or the value set computation problem.

Several approaches to solve the template generation problem exist. However, these existing template generation methods (with the exception of grid-based ones) are restricted in scope to particular kinds of correlations and dependencies in the system parameters: independent parameters (Bailey, Panzer \& Gu, 1988; Bailey \& Panzer, 1988; Fialho, Pande \& Nataraj, 1992; Hwang \& Chen,
1995; Lan, 1994); affine parametric dependency (Bailey \& Hui, 1989; Bartlett, 1990; Fu, 1990); multi-linear dependency (Bhattacharyya et al., 1995); and special nonlinear dependency (Barmish \& Tempo, 1995). Further, in these methods there are inherent restrictions to transfer functions of particular forms, such as rational, tree-structured decomposable (Barmish, Ackermann \& Hu, 1990; Ackermann \& Sienel, 1991; Eszter \& Pena, 1994); factored real form (Gutman, Baril \& Neumann, 1994), and other forms (Fu, Dasgupta \& Blondel, 1995; Isogai, Ohta \& Haneda, 1993; Ohta, 1996; Ohta, Gong \& Haneda, 1994). A few of these algorithms (Lasky \& Ravani, 1997) carry out convex hull approximations of the template, which could be quite conservative in cases where the actual template is non-convex. Grid-based methods (Cohen, Nordin \& Gutman, 1995; Horowitz, 1991), are applicable with no known restrictions on the kind of parametric dependency or form of transfer function. Unfortunately, a limitation with any grid-based method is that it is potentially risky, in the sense that some critical points of the templates could be missed due to the very nature of the grid process.

This paper presents a template generation algorithm based on interval analysis methods. The merits of the proposed algorithm are as follows. (i) The algorithm has a vast scope - it is applicable to any transfer function whose magnitude and phase are continuous 
functions of the uncertain system parameters. These continuous functions may be computable as explicit formulas, recursively, via numerical algorithms, or computer codes. Further, these functions could involve any combination and compositions of the elementary arithmetic operations and unary functions (including all pre-declared functions of a programming language, such as sin, cos, exp, log, sqrt, power, and arctan). (ii) The algorithm can generate, in theory, templates of arbitrary accuracy (in practice, the accuracy is limited by the available machine precision and memory storage). (iii) The algorithm, when implemented on an interval arithmetic compiler, generates a so-called safe and reliable template that includes all the actual template points at the same frequency, despite all kinds of computational errors, such as roundoff and truncation. This key aspect guarantees that the resulting controller design is indeed valid over the entire plant set. (iv) If the magnitude and phase functions are continuously differentiable, then, this property can be exploited to considerably speed up the algorithm, and to obtain a significantly smaller set of solution rectangles. (v) No rational function approximation of the non-rational transfer function is performed in the algorithm.

This paper is organized as follows. Section 2 gives the essentials of interval mathematics relevant to the work. Section 3 presents the template generation algorithm. Section 4 gives theoretic properties of the algorithm. An illustrative example follows in Section 5. In Section 6, the conclusions of the work are given.

\section{Real interval mathematics}

This section collects some preliminaries of interval analysis. A real interval is a closed and bounded set of real numbers $X:=[\underline{X}, \bar{X}]:=\{x \in \mathfrak{R}: \underline{X} \leq x \leq \bar{X}\}$ where, $X$ and $\bar{X}$ are the lower and upper endpoints of the interval $X$. The set of all real intervals is denoted by $I \Re$. Two intervals are equal if their corresponding endpoints are equal. The intersection of two intervals $X$ and $Y$ is empty, $X \cap Y=\emptyset$, if either $X>\bar{Y}$ or $Y>\bar{X}$. Else, the intersection of $X$ and $\bar{Y}$ is again an interval $X \cap Y:=[\max (\underline{X}, \underline{Y}), \min (\bar{X}, \bar{Y})]$. The set inclusion: $X \subseteq Y$ if and only if $Y \leq X$ and $\bar{X} \leq \bar{Y}$. Further, the width of an interval $X$ is defined as $w(X):=\bar{X}-X$, and the mean as $m(X):=(X+\bar{X}) / 2$.

An interval vector is a vector whose elements are intervals. The set of all $n$-dimensional real interval vectors is denoted by $I \Re^{n}$. The notation $X:=$ $\left(X_{1}, \ldots, X_{n}\right), X \in I \Re^{n}$ is used. The relations $\epsilon,=$, and $\subseteq$, union, intersection, and mean are all defined component wise. 'Box' is a synonym for an interval vector, and 'rectangle' for a 2-dim interval vector.

In addition to the elementary arithmetic operations, there are further common, mostly unary, operations on intervals (Moore, 1979):
Definition 2.1. Let $r(x)$ be a continuous unary operation defined on $\mathfrak{R}$. Then, a unary operation on $I \mathfrak{R}$ is defined as $r(X):=\left[\min _{x \in X}\{r(X)\}, \max _{x \in X}\{r(X)\}\right]$.

\subsection{Interval extension and range enclosures}

Definition 2.2. Let $f: \Re^{n} \rightarrow \Re^{m}$ be a function defined over a box $X \in I \Re^{n}$. Then, the range of $f$ over $X$ is denoted as $\bar{f}(X)$. That is, $\bar{f}(X):=\{f(x): x \in X\}$.

Definition 2.3 (Interval extension). A function $F: I \Re^{n} \rightarrow I \Re^{m}$ is said to be an interval extension of function $f: \mathfrak{R}^{n} \rightarrow \mathfrak{R}^{m}$ provided $\bar{f}(X) \subseteq F(X)$ for all boxes $X \in I \Re^{n}$ within the domain of $F$.

Definition 2.4 (Natural interval extension). If $f: \mathfrak{R}^{n} \rightarrow \mathfrak{R}^{m}$ is a function computable as an expression, algorithm, or computer program involving the four elementary arithmetic operations interspersed with evaluations of unary functions, then a natural interval extension of $f$, whose value over a box $X$ is denoted by $F(X)$, is obtained by replacing each occurrence of each component $x_{i}$ of $x$ by the corresponding interval component $X_{i}$ of $X$, by executing all operations according to standard interval arithmetic rules, and by computing the exact ranges of the unary functions as in Definition 2.1.

Definition 2.5. An interval extension $F$, defined over a domain $X$, is said to be inclusion monotonic if $Y \subseteq X \Rightarrow F(Y) \subseteq F(X)$.

Two key properties of interval extensions are

Theorem 2.1 ((Moore, 1979) (Inclusion monotonicity)). Natural interval extensions are inclusion monotonic.

Theorem 2.2 ((Moore, 1979) (Inclusion property)). Let $F(X)$ denote a natural interval extension of $f(x)$. Then, $\bar{f}(X) \subseteq F(X)$.

\subsection{Interval topology}

Definition 2.6. Let $A, B$ be compact, non-empty subsets of $\mathfrak{R}^{n}$ and $x \in \mathfrak{R}^{n}$. Define

$$
\begin{aligned}
& d_{0}(x, B):=\min _{b \in B}\|x-b\|_{2}, \quad d_{0}(A, B):=\max _{a \in A} d_{0}(a, B), \\
& d(A, B):=\max \left\{d_{0}(A, B), d_{0}(B, A)\right\} .
\end{aligned}
$$

The Hausdorff-distance $d$ is a metric for the sets of compact non-empty subsets of $\mathfrak{R}^{n}$. With this metric, a topology is defined, and convergence, etc., can be defined in the usual manner. In particular, the following definition can be made:

Definition 2.7. A sequence $\left(X_{k}\right)_{k=1}^{\infty}$ of interval vectors $X_{k} \in I \Re^{n}$ is said to converge to a point $z \in \mathfrak{R}^{n}$ if $d\left(X_{k}, z\right) \rightarrow 0$ as $k \rightarrow \infty$. 


\subsection{Interval linear systems}

Consider the rectangular interval system $A X=B$, where $A \in I \mathfrak{R}^{m \times n}$ and $B \in I \mathfrak{R}^{m}, m \leq n$. The solution set of this system is that set $\sum(A, B) \subseteq \mathfrak{R}^{n}$ such that if $x \in \sum(A, B)$, there exists an $\widetilde{A} \in A$ and a $\widetilde{B} \in B$ such that $\tilde{A} x=\widetilde{B}$. The so-called Generalized Gauss-Seidel method (GGS) has been employed in Neumaier (1988) to find such a solution set (preconditioning of the system is strongly suggested). The reader is referred to Neumaier (1988) for details of the GGS method. The following adaptation of the result in Neumaier (1990) is required in the sequel.

Theorem 2.3. Consider the interval linear system $A X=B$ where $A \in I \mathfrak{R}^{m \times n}$ and $B \in I \mathfrak{R}^{m}, m \leq n$. Let $\sum(A, B)$ denote the solution set of this system. Then

$\sum(A, B) \cap X^{C} \subseteq \sum(C A, C B) \cap X^{C} \subseteq X_{\mathrm{GGS}}$

where, the preconditioning matrix $C \in \mathfrak{R}^{m \times m}$ is an approximate inverse of a matrix composed of $m$ linearly independent columns of some $\tilde{A} \in A, X_{\mathrm{GGS}}$ is the solution obtained using GGS for the preconditioned interval system, and $X^{C}$ is the initial interval vector (box) in which the solution is sought.

\section{Algorithm for template generation}

Consider a system represented by the transfer function $g(s, \lambda)$, where $\lambda=\left\{\lambda_{1}, \ldots, \lambda_{l}\right\}$ is a real vector of the fundamental system parameters, and $s$ is the Laplace variable. Suppose the parameters $\lambda_{i}$ vary independently over given real intervals $\Lambda_{i}^{0}$, so that $\Lambda^{0}:=\left\{\Lambda_{1}^{0}, \ldots, \Lambda_{l}^{0}\right\}$ is a box.

Definition 3.1 (In above notation). The angle and magnitude functions for the transfer function $g(s, \lambda)$ are defined as

$h_{\text {ang }}(\lambda):=\arg \{g(s=j \omega, \lambda)\}, \quad h_{\text {mag }}(\lambda):=|g(s=j \omega, \lambda)|$

where, $j=\sqrt{-1}$, and $\omega$ is the frequency variable (whose value is fixed). Further, define $h(\lambda):=\left(h_{\text {ang }}(\lambda), h_{\text {mag }}(\lambda)\right)$. Then, the set $\mathscr{G}:=\bar{h}\left(\Lambda^{0}\right):=\left\{h(\lambda), \lambda \in \Lambda^{0}\right\}$ is called as the template of $g(s, \lambda)$ at the given $\omega$.

Definition 3.2. Let $\Lambda$ denote the interval vector variable corresponding to the real vector variable $\lambda$. Then, $H_{\text {ang }}(\Lambda)$ will denote a natural interval extension of $h_{\text {ang }}$ over $\Lambda$. Similarly for $H_{\text {mag }}(\Lambda)$.

Definition 3.3. Let $n=l+2$, where $l$ is the length of the $\lambda$ vector. Define the box $D \in I \mathfrak{R}^{n}$ as follows:

$D:=\left(H_{\text {ang }}\left(\Lambda^{0}\right), H_{\text {mag }}\left(\Lambda^{0}\right), \Lambda^{0}\right)$.
Definition 3.4. Let $x=\left(x_{1}, x_{2}, \lambda\right) \in D$, and define the function

$f(x):=\left(x_{1}-h_{\mathrm{ang}}(\lambda), x_{2}-h_{\mathrm{mag}}(\lambda)\right)$.

Further, let $X$ denote the interval vector variable corresponding to $x$. Then, $F(X)$ will denote a natural interval extension of $f$ over $X$.

Definition 3.5. Let $y:=\left(x_{1}, x_{2}\right)$, so that $x=(y, \lambda)$. In terms of corresponding interval variables, let $Y:=\left(X_{1}, X_{2}\right)$ so that $X=(Y, \Lambda)$. Then, $Y$ will be called as the 'constituent rectangle' of the subbox $X$.

Definition 3.6. Suppose $f$ is differentiable on $D$. Then, the Jacobi matrix (arranged as a $2 \times n$ matrix) of $f(x)$ in (3) will be denoted by $f^{\prime}(x)$; further, $F^{\prime}(X)$ will denote any interval Jacobi matrix whose components are natural interval extensions over $X$ of corresponding components of $f^{\prime}$.

Throughout this work, the following will be assumed.

Assumption 1. $f$ is continuous, with its natural interval extension $F$ well-defined, on D.

The template generation algorithm is presented next.

Algorithm (Template Generation Algorithm).

Inputs: An expression for the transfer function $g(s, \lambda), \Lambda^{0}$, $\omega$, and a parameter $\varepsilon_{x}$ specifying the maximum width of each solution constituent rectangle.

Output: The approximating template $\mathscr{G}^{\text {alg }}$ constructed in step 3a below.

Note: The algorithm is to be executed in the order given below, except when otherwise indicated.

BEGIN Algorithm

1. (Symbolic manipulation phase)

(a) From the transfer function expression $g(s, \lambda)$, obtain the angle and magnitude functions $h_{\text {ang }}(\lambda)$, $h_{\text {mag }}(\lambda)$ in $(1)$.

(b) Find the natural interval extensions $H_{\text {ang }}(\Lambda), H_{\text {mag }}(\Lambda)$ from the expressions for $h_{\text {ang }}(\lambda), h_{\text {mag }}(\lambda)$, respectively.

(c) Form the function $f(x)$ in (3), and find the natural interval extension $F(X)$ from the expression for $f(x)$.

2. (Initialization phase)

(a) Compute $D$ in (2).

(b) Set: $X^{0} \leftarrow D$.

(c) Set: $k \leftarrow 0$.

(d) Initialize lists: $\mathscr{L}^{\text {sol }} \leftarrow\{\}, \mathscr{L} \leftarrow\left\{X^{0}\right\}$.

3. (Start a new iteration)

(a) If $\mathscr{L}$ is empty, construct $\mathscr{G}^{\text {alg }}:=\bigcup_{Y_{i} \in \mathscr{L}^{\text {sol }}} Y_{i}$, and EXIT.

(b) $k \leftarrow k+1$ 
(c) Choose the first box from $\mathscr{L}$, call it $X$, and delete $X$ from $\mathscr{L}$.

4. (Test phase and solution list)

(a) (Exclusion test) Evaluate $F(X)$. If $0 \notin F(X)$, then discard $X$ and return to Step 3a.

(b) (GGS Step. Executed only if $f$ is continuously differentiable, and natural interval extension $F^{\prime}$ is well-defined, on $D$ ) Using automatic differentiation (Moore, 1979) evaluate the interval Jacobi matrix $F^{\prime}(X)$. Next, using GGS, find the solution set $X_{\mathrm{GGS}}$ of the homogenous interval linear equation $A \tilde{X}=0$, where

$$
\begin{aligned}
& A=\left(F^{\prime}(X) \vdots f^{\mathrm{T}}(\tilde{z})\right), \quad \tilde{X}=(X-\tilde{z} \vdots 1)^{\mathrm{T}}, \\
& \tilde{z}=m(X)
\end{aligned}
$$

If GGS returns an empty solution set, then discard $X$ and return to Step 3a. Else, set $X \leftarrow X_{\mathrm{GGS}}^{\mathrm{T}}+\tilde{z}$.

(c) If $w(X)<\varepsilon_{x}$, enter $Y$ into $\mathscr{L}^{\text {sol }}$ (note that $X=(Y, \Lambda))$ and return to Step 3a.

5. (Bisection phase)

(a) Choose a coordinate direction parallel to which $X$ has an edge of maximum width.

(b) Bisect $X$ along the above chosen coordinate direction to get two sub-boxes $X^{A}, X^{B}$ such that $X=X^{A} \cup X^{B}$.

(c) Enter $X^{A}, X^{B}$ into $\mathscr{L}$ such that the widths of boxes in $\mathscr{L}$ do not increase.

6. (End current iteration) Return to Step 3a.

END Algorithm.

Remark 3.1. The generality of the algorithm is emphasized. Assumption 1 means that the algorithm accepts as functional parameters magnitude and phase functions that are continuous and have natural interval extensions well-defined on $D$. Now, such extensions can be constructed for any magnitude or phase function expressible as combination and compositions of the elementary arithmetic operations and unary functions (Moore, 1979, pg. 3). The unary functions include absolute value, square, square root, exponential function, power function , logarithmic functions, trigonometric and inverse trigonometric functions, and hyperbolic and inverse hyperbolic functions. Other functions may also be included in this list, provided they are continuous on each closed interval on which they are defined. Hence, one can obtain natural interval extensions for virtually any magnitude or phase function that can be represented with a computer program (Kearfott, 1996, pg. 12).

Remark 3.2. The algorithm as given above assumed the availability of explicit functional expressions for $h_{\text {ang }}(\lambda), h_{\text {mag }}(\lambda)$, and $f(x)$. This was done only for presentational convenience and is not necessary. Alternate representations for $h_{\text {ang }}(\lambda), h_{\text {mag }}(\lambda)$, and $f(x)$, such as recursive ones or computer codes, may be used. The construction of natural interval extensions for these alternate representations is straightforward, and runs completely automatically with the help of interval compilers (Ratschek, 1985); similar remarks apply to the task of automatic differentiation in step $4 \mathrm{~b}$.

Remark 3.3. If $f$ is continuously differentiable on $D$, then this property can be used to speed up the algorithm through execution of Step 4b. A considerably smaller set of solution rectangles is also usually obtained in this case. Further, in this step, it is usually more effective to perform two sweeps of the GGS method (Neumaier, 1988) and use an interval slope matrix instead of an interval Jacobi matrix (Kearfott 1996).

Remark 3.4. For subsequent robustness analysis and controller design purposes, a more wieldy representation of the template (in terms of a reduced number of rectangles) can be obtained by applying steps 7-9 of the algorithm in Sardar and Nataraj (1997) to $\mathscr{G}^{\text {alg }}$.

\section{Theoretic properties of algorithm}

The below lemma says that the algorithm is initialized with its initial list $\mathscr{L}$ containing a constituent rectangle that encloses the template.

Lemma 4.1. Let $X^{0}$ be as in Step $2 \mathrm{~d}$ of the algorithm, and $Y^{0}$ be the constituent rectangle of $X^{0}$. Then, $\mathscr{G} \subseteq Y^{0}$.

Proof. In Step $2 \mathrm{~d}$ of the algorithm, $X^{0} \leftarrow D=\left(H_{\text {ang }}\left(\Lambda^{0}\right)\right.$, $\left.H_{\text {mag }}\left(\Lambda^{0}\right), \Lambda^{0}\right)$, so $Y^{0} \leftarrow\left(H_{\text {ang }}\left(\Lambda^{0}\right), H_{\text {mag }}\left(\Lambda^{0}\right)\right)$. By the inclusion property (Theorem 2.2$), \bar{h}\left(\Lambda^{0}\right) \subseteq\left(H_{\text {ang }}\left(\Lambda^{0}\right), H_{\text {mag }}\left(\Lambda^{0}\right)\right)$. Hence, $\mathscr{G}:=\bar{h}\left(\Lambda^{0}\right) \subseteq Y^{0}$.

The below lemma justifies Step $4 \mathrm{a}$ in the algorithm.

Lemma 4.2. Let $X \subseteq X^{0}$ be a subbox, where $X^{0}$ is as in Step $2 \mathrm{~b}$ of the algorithm. Suppose, $0 \notin F(X)$. Then, $X$ can be discarded in the algorithm.

Proof. By hypothesis, $0 \notin F(X)$. Then, from Definitions 3.4 and $3.5, Y \cap H(\Lambda)=\emptyset$. By the inclusion property in Theorem 2.2, $\bar{h}(\Lambda) \subseteq H(\Lambda)$. Thus, $Y \cap \bar{h}(\Lambda)=\emptyset$, i.e., the constituent rectangle $Y$ of $X$ does not contain any template points. Hence, $X$ is irrelevant in the search for template points, and can be discarded.

The below two lemmas justify (if executed) Step 4b. in the algorithm.

Lemma 4.3 (If Step $4 \mathrm{~b}$ is executed). Let $X \subseteq X^{0}$ be $a$ subbox, and suppose that the GGS method applied to $X$ 
in Step $4 \mathrm{~b}$ returns an empty solution set, i.e., $X_{\mathrm{GGS}}=\emptyset$. Then, $X$ can be discarded in the algorithm.

Proof. Suppose $x^{*} \in X$ is such that $f\left(x^{*}\right)=\left(f_{1}\left(x^{*}\right)\right.$, $\left.f_{2}\left(x^{*}\right)\right)=0$. Let $\tilde{z}=m(X)$. Then, the mean value theorem of calculus gives

$0=f_{1}\left(x^{*}\right)=f_{1}(\tilde{z})+f_{1}^{\prime}\left(\zeta_{1}\right)\left(x^{*}-\tilde{z}\right)^{\mathrm{T}}, \quad \zeta_{1} \in X$,

$0=f_{2}\left(x^{*}\right)=f_{2}(\tilde{z})+f_{2}^{\prime}\left(\zeta_{2}\right)\left(x^{*}-\tilde{z}\right)^{\mathrm{T}}, \quad \zeta_{2} \in X$.

From Theorem 2.2, $f_{1}^{\prime}\left(\zeta_{1}\right) \in F_{1}^{\prime}(X), f_{2}^{\prime}\left(\zeta_{2}\right) \in F_{2}^{\prime}(X)$. Thus, $\left(x^{*}-\tilde{z}\right)^{\mathrm{T}}$ must lie in the solution set to

$F^{\prime}(X)(X-\tilde{z})^{\mathrm{T}}=-f^{\mathrm{T}}(\tilde{z})$.

By Theorem 2.3, $X_{\mathrm{GGS}}$ got using the GGS method encloses the solution set of (4). Hence, any root $x^{*}$ of $f$ within $X$ must be within $\tilde{z}+X_{\mathrm{GGS}}^{\mathrm{T}}$. From this, it follows that if $X_{\mathrm{GGS}}=\emptyset$, then there are no roots of $f$ within $X$, implying that no template points lie in the constituent rectangle $Y$ of $X$. Hence, $X$ can be discarded in the search for template points.

Lemma 4.4 (If Step 4b is executed). Let $X \subseteq X^{0}$ be a subbox, and suppose that the GGS method applied to $X$ in Step $4 \mathrm{~b}$ returns a non-empty solution set, i.e., $X_{\mathrm{GGS}} \neq \emptyset$. Then, the constituent rectangle (of the GGS solution) that is produced retains any template points present in the constituent rectangle $Y$ of $X$.

Proof. Continuing the proof in the preceding lemma for the other case $X_{\mathrm{GGS}} \neq \emptyset$, it follows that any template point present in the constituent rectangle $Y$ of $X$ must also be present in the constituent rectangle of $\tilde{z}+X_{\mathrm{GGS}}^{\mathrm{T}}$, proving the assertion of this lemma.

\subsection{Convergence}

Its assumed in this sub-section that the termination criterion in the algorithm can never be satisfied (i.e. $\varepsilon_{x}=0$ ) so that the convergence properties can be studied. It is also assumed for the same reason that the list sizes are not a limitation. First, some further quantities are defined.

Definition 4.1. Let $\mathscr{L}_{k}$ denote the list $\mathscr{L}$ present at the start of the $k$ th iteration of the algorithm, and denote the $i$ th box of this list as $X_{k i}=\left(Y_{k i}, \Lambda_{k i}\right)$. Define the following unions

$U_{k}=\bigcup_{Y_{k i} \in \mathscr{L}_{k}} Y_{k i}, \quad V_{k}=\bigcup_{\Lambda_{k i} \in \mathscr{L}_{k}} \Lambda_{k i}, \quad W_{k}=\bigcup_{X_{k i} \in \mathscr{L}_{k}} X_{k i}$.

Lemma 4.5. $\mathscr{G}$ is a compact set in $\mathfrak{R}^{2}$.

Proof. By Assumption 1, $h$ is continuous on $D$. Clearly, the parameter box $\Lambda^{0}$ is compact. As the image of a con- tinuous mapping on a compact set is compact, so $\bar{h}\left(\Lambda^{0}\right)$, i.e., $\mathscr{G}$, is compact.

Similarly, it can be shown that

Lemma 4.6. The unions $U_{k}, V_{k}, W_{k}$, are compact.

Lemma 4.7. Let $U_{k}$ be as in (5). Then, $\mathscr{G} \subseteq \bigcap_{1}^{\infty} U_{k}$.

Proof. It suffices to show that $\mathscr{G} \subseteq U_{k}$ for any $k$. The assertion of the lemma then follows. Consider the algorithm with $k=1$. Firstly, note that, by Lemma 4.1, all the template points are contained in $Y^{0}$, i.e., $\mathscr{G} \subseteq U_{1}$. By Lemma 4.2, the discarding process in Step 4a of the algorithm does not delete any template point contained in $Y^{0}$. Further, if Step $4 \mathrm{~b}$ is executed, then, by Lemma 4.4, the GGS method also does not delete any template point contained in $Y^{0}$. Moreover, no template point in $Y^{0}$ can be lost in the bisection phase (because both the sub-boxes resulting from bisection are entered into the list). Thus, at the end of the first iteration, all the template points are retained in $U_{2}$, i.e., $\mathscr{G} \subseteq U_{2}$. By induction on $k$, we have $\mathscr{G} \subseteq U_{k}$ for any $k \Rightarrow \mathscr{G} \subseteq \cap_{1}^{\infty} U_{k}$.

Remark 4.1. From the above lemma, it can be seen that the unions $U_{k}, V_{k}, W_{k}$, are non-empty compact sets, for any $k$. Further, $\bigcap_{1}^{\infty} U_{k}$ and $\bigcap_{1}^{\infty} V_{k}$ are non-empty sets.

Lemma 4.8. Let $U_{k}$ be as in (5). Then, the sequence $\left(U_{k}\right)_{k=1}^{\infty}$ has the property $U_{1} \supseteq U_{2} \supseteq U_{3} \cdots$.

Proof. In the first iteration $(k=1)$ of the algorithm, the box $X^{0}$ is picked from the list $\mathscr{L}_{1}$, (some) irrelevant portions of $X^{0}$ removed in the GGS step (if Step $4 \mathrm{~b}$ is executed), and the remaining bisected; the boxes resulting from the bisection process then replace $X^{0}$ in $\mathscr{L}_{1}$, giving the list $\mathscr{L}_{2}$ (state of the list at $k=2$ ). The proof is completed by induction on $k$.

Lemma 4.9. Let $w_{k}$ denote the maximum width of boxes of the kth list $\mathscr{L}_{k}$ generated by the algorithm. Then, $w_{k} \rightarrow 0$ as $k \rightarrow \infty$

Proof. Follows from the lemma in Ratschek (1985).

Lemma 4.10. Let $X$ and $F(X)$ be as in Definition 3.4. Then, $w(F(X)) \rightarrow 0$ as $w(X) \rightarrow 0$

Proof. By Theorem 1.5 in Kearfott (1996), all natural interval extensions are inclusion functions of first-order, that is, there is a constant $\alpha>0$ independent of the box $X$ such that

$w(F(X))-w(\bar{f}(X)) \leq \alpha w(X)$

for all boxes $X \subseteq D$ with $w(X)$ sufficiently small. Since, by Assumption $1, f$ is continuous on $D, w(\bar{f}(X)) \rightarrow 0$ as 
$w(X) \rightarrow 0$. Together with (6), this leads to the assertion of the lemma.

Definition 4.2. Let $f(x)$ be as in (3), and $X^{0}$ be as in Step $2 \mathrm{~b}$ of the algorithm. Define the solution set $\sum\left(f, X^{0}\right)$ as

$\sum\left(f, X^{0}\right):=\left\{x: f(x)=0, x \in X^{0}\right\}$.

Lemma 4.11. Let $W_{k}$ be as in (5), and $\sum\left(f, X^{0}\right)$ as in (7). Then, $\cap_{1}^{\infty} W_{k} \subseteq \sum\left(f, X^{0}\right)$.

Proof. Let $x \in W_{k} \forall k$. It is to be shown that $x \in \sum\left(f, X^{0}\right)$. Since $x \in W_{k} \forall k$, it follows first that, for any $k$, an item $X_{k}^{\prime}$ must occur in the list $\mathscr{L}_{k}$ such that $x \in X_{k}^{\prime}$. That is,

$x \in X_{k}^{\prime}$ for all $k$.

By Lemma 4.9.

$w\left(X_{k}^{\prime}\right) \rightarrow 0 \quad$ as $k \rightarrow \infty$.

From (8), (9), and Definition 2.7 it follows that the sequence $\left(X_{k}^{\prime}\right)_{k=1}^{\infty}$ tends to $x$ :

$X_{k}^{\prime} \rightarrow x \quad$ as $k \rightarrow \infty$.

Since $f$ is continuous by Assumption 1, this gives

$\bar{f}\left(X_{k}^{\prime}\right) \rightarrow f(x)$ as $k \rightarrow \infty$.

By the inclusion property in Theorem 2.2,

$f(x) \in \bar{f}\left(X_{k}^{\prime}\right) \subseteq F\left(X_{k}^{\prime}\right)$.

Moreover, from (9) and Lemma 4.10

$w\left(F\left(X_{k}^{\prime}\right)\right) \rightarrow 0 \quad$ as $k \rightarrow \infty$.

From (10), (11), and Definition 2.7

$F\left(X_{k}^{\prime}\right) \rightarrow f(x)$ as $k \rightarrow \infty$.

Further, since $X_{k}^{\prime}$ is not yet discarded

$0 \in F\left(X_{k}^{\prime}\right)$ for any $k$.

From (11), (13)

$F\left(X_{k}^{\prime}\right) \rightarrow 0$ as $k \rightarrow \infty$.

Comparing (12) and (14) gives

$f(x)=0$.

That is, $x \in \sum\left(f, X^{0}\right)$.

Lemma 4.12. Let $U_{k}$ be as in (5). Then, $\cap_{1}^{\infty} U_{k} \subseteq \mathscr{G}$.

Proof. Consider the $k$ th list $\mathscr{L}_{k}$ generated by the algorithm. Denote the $i$ th box of this list as $X_{k i}=\left(Y_{k i}, \Lambda_{k i}\right)$. From (5),

$$
\begin{aligned}
W_{k}=\bigcup_{X_{k i} \in L_{k}} X_{k i} & =\bigcup_{Y_{k i}, \Lambda_{k i} \in L_{k}}\left(Y_{k i}, \Lambda_{k i}\right) \\
& =\left(\bigcup_{Y_{k i} \in L_{k}} Y_{k i}, \bigcup_{\Lambda_{k i} \in L_{k}} \Lambda_{k i}\right) .
\end{aligned}
$$

Therefore, $W_{k}=\left(U_{k}, V_{k}\right)$. This gives (by Remark 4.1, the intersections are non-empty)

$\bigcap_{1}^{\infty} W_{k}=\bigcap_{1}^{\infty}\left(U_{k}, V_{k}\right)=\left(\bigcap_{1}^{\infty} U_{k}, \bigcap_{1}^{\infty} V_{k}\right)$.

From (7),

$$
\begin{aligned}
\sum\left(f, X^{0}\right) & =\left\{x: f(x)=0, x \in X^{0}\right\} \\
& =\left\{(y, \lambda): y=h(\lambda), y \in Y^{0}, \lambda \in \Lambda^{0}\right\} \\
& =\left\{(h(\lambda), \lambda), \lambda \in \Lambda^{0}\right\} .
\end{aligned}
$$

By Lemma 4.11, $\bigcap_{1}^{\infty} W_{k} \subseteq \sum\left(f, X^{0}\right)$. From (15) and (16),

$$
\begin{aligned}
& \left(\bigcap_{1}^{\infty} U_{k}, \bigcap_{1}^{\infty} V_{k}\right) \\
& \subseteq\left\{(h(\lambda), \lambda), \lambda \in \Lambda^{0}\right\} \Rightarrow \bigcap_{1}^{\infty} U_{k} \\
& \quad \subseteq\left\{h(\lambda), \lambda \in \Lambda^{0}\right\}=\bar{h}\left(\Lambda^{0}\right)=: \mathscr{G} .
\end{aligned}
$$

Lemma 4.13. Let $U_{k}$ be as in (5). Then, $d\left(U_{k}, \mathscr{G}\right) \rightarrow 0$ as $k \rightarrow \infty$.

Proof. By Lemma 4.8, the unions $U_{k}$ form a chain

$U_{1} \supseteq U_{2} \supseteq U_{3} \cdots$.

From Lemmas 4.7 and 4.12

$\mathscr{G}=\bigcap_{1}^{\infty} U_{k}$.

Now, (17) and (18) together imply that the unions $U_{k}$ converge to $\mathscr{G}$, i.e., $d\left(U_{k}, \mathscr{G}\right) \rightarrow 0$ as $k \rightarrow \infty$.

The following theorem gives the convergence property of the algorithm.

Theorem 4.14. The collection of solution rectangles $Y$ (generated in the list $\mathscr{L}$ of the algorithm) converges, from without, to the template $\mathscr{G}$.

Proof. Let $A, B \in \mathfrak{R}^{2}$ be any two non-empty compact subsets. From Definition 2.6, it is clear that $d(A, B)=0$ only if $A=B$. Therefore, it follows from Lemma 4.13 that convergence of unions $U_{k}$ to the template $\mathscr{G}$ via $d$, i.e., $d\left(U_{k}, \mathscr{G}\right) \rightarrow 0$ as $k \rightarrow \infty$, means that $U_{k}$ converges to $\mathscr{G}$. That this convergence is from without follows from the property $\mathscr{G} \subseteq U_{k}$, for any $k$ (cf. proof of Lemma 4.7).

\subsection{Finite termination}

It is next shown that for $\varepsilon_{x}>0$, the algorithm terminates in a finite number of iterations. 
Theorem 4.15. The algorithm terminates after at most $\chi^{n}-1$ iterations, where $\chi=w\left(X^{0}\right) / \varepsilon_{x}$.

Proof. First note that, after n.p successive bisections, where $n \geq 2$ (cf. Definition 3.3), $w(X) \leq 2^{-p} w\left(X^{0}\right)$. Thus, after at most $\Xi:=n \log _{2}\left(w\left(X^{0}\right) / \varepsilon_{x}\right)$ successive bisections the processing of a subbox is completed (because then $\left.w(X) \leq \varepsilon_{x}\right)$. The algorithm produces a binary tree whose nodes are the regions obtained through successive bisections. The root of this tree is the node corresponding to the original region $X^{0}$. The depth of this tree is equal to the maximum number of successive bisections, which is at most $\Xi$. The maximum total number of bisections occurs for a balanced tree, and is equal to the number of internal nodes in this tree. From Tucker (1980), this maximum number would be equal to $2^{\Xi}-1=\chi^{n}-1$, where $\chi=w\left(X^{0}\right) / \varepsilon_{x}$.

Theorem 4.16. $\mathscr{G} \subseteq \mathscr{G}^{\text {alg }}$.

Proof. Let $\mathscr{L}_{k}^{\text {sol }}$ denote the list $\mathscr{L}^{\text {sol }}$ present at the end of the $k$ th iteration of the algorithm. By Theorem 4.15, for a given $\varepsilon_{x}>0$, the algorithm terminates at a finite iteration number, denoted $\alpha$. Noting that for $\varepsilon_{x}>0$ case, $Y$ is entered when appropriate into $\mathscr{L}^{\text {sol }}$ in step $4 \mathrm{c}$, and proceeding similarly as in the proof of Lemma 4.7, it can be shown that $\mathscr{G} \subseteq\left(\cup_{Y_{i} \in \mathscr{L}_{k-1}^{\text {sol }}} Y_{i}\right) \cup U_{k}$, for any $k$. Combining this with the fact that the algorithm terminates when $\mathscr{L}$ is found empty at the end of the $\alpha$ th iteration, gives $\mathscr{G} \subseteq \cup_{Y_{i} \in \mathscr{L}_{2}^{\text {sol }}} Y_{i}=: \mathscr{G}^{\text {alg }}$.

\subsection{Safety and reliability}

Definition 4.3 (Safe template). Let $\omega$ be a fixed frequency, and $g(s, \lambda)$ be as given above. Let $\mathscr{G}$ denote the actual template, and $\mathscr{G}^{\text {alg }}$ denote the template generated by an appropriate algorithm, both at $\omega$. Then, $\mathscr{G}^{\text {alg }}$ is said to be a safe template if $\mathscr{G} \subseteq \mathscr{G}^{\text {alg }}$. Else, $\mathscr{G}^{\text {alg }}$ is said to be an unsafe template.

A safe template, therefore, includes the frequency responses of the entire plant set at the same $\omega$. This inclusion property of a safe template guarantees that the resulting control design would be valid over the entire plant set. This important property is shown to hold for the template generated by the proposed algorithm:

Theorem 4.17 (Safety). Let $\omega$ be a given frequency. Then, the generated template $\mathscr{G}^{\text {alg }}$ is a safe template as per Definition 4.3.

Proof. From Theorem 4.16, $\mathscr{G} \subseteq \mathscr{G}^{\text {alg }}$. Therefore, by Definition $4.3, \mathscr{G}^{\text {alg }}$ is a safe template.

In the developments given above, exact interval arithmetic was assumed in computing the results. Exact arithmetic assumes infinite computing precision, and so is an idealized arithmetic. When such an arithmetic is implemented on a computer, then the endpoints of the intervals must be approximated by floating point or machine numbers. Each floating point operation is subject to error, and the default rounding may result in loss of rigor. Therefore, any theoretical results may be found invalidated in practice (on computers). An error analysis to account for these effects is rather troublesome.

Machine interval arithmetic provides a framework to generate reliable results taking into account all kinds of computational errors. In computations using machine interval arithmetic, values of input parameters are outwardly rounded to become intervals containing the true value. In addition, using outwardly rounded interval arithmetic throughout the computations assures that the computed result contains the actual range despite the presence of rounding errors. This outward rounding process is used together with the exact interval arithmetic rules to define a machine interval arithmetic.

Machine interval arithmetic can provide mathematically rigorous results from floating point operations on computers (Moore, 1979). The reader is referred to Klatte, Kulisch, Neaga, Ratz and Ullrich (1993) for details of machine interval arithmetic. Its utility for computing reliable results is evident from the following

Theorem 4.18 (Inclusion property of machine interval arithmetic (Moore, 1979)). Let $f$ be a real function of $x \in \mathfrak{R}^{n}$, and let $f(x)$ be an expression for $f$. Assume that the interval extensions $F(X)$ and $F^{\diamond}(X)$ (obtained using exact and machine interval arithmetic) are well-defined for the interval $X$ corresponding to $x$. Then, it follows that $\bar{f}(X) \subseteq F(X) \subseteq F^{\diamond}(X)$, where $\bar{f}(X)$ denotes the range of fover $X$.

Definition 4.4 (Reliable template). A template generated by an appropriate algorithm is said to be reliable, if it has been computed taking into account all kinds of computational errors, such as round-off, truncation, and approximation errors.

In practice, a machine interval arithmetic implementation of the proposed algorithm is used to account for all kinds of computational errors. The below theorem shows the reliability of the template results generated by a machine interval arithmetic implementation of the proposed algorithm.

Theorem 4.19 (Reliability). Let $\omega$ be a given frequency. Let $\left.\mathscr{G}^{\mathrm{alg}}\right|_{\text {mia }}$ denote the template generated by a machine interval arithmetic implementation of the algorithm. Then, $\left.\mathscr{G} \subseteq \mathscr{G}^{\text {alg }} \subseteq \mathscr{G}^{\mathrm{alg}}\right|_{\text {mia }}$.

Proof. Follows from Theorems 4.16 and 4.18. 


\section{Illustrative example}

The following example demonstrates the capability of the algorithm to deal with a wide class of transfer functions. Consider the following transfer function:

$$
\begin{aligned}
g(s, \lambda)= & \frac{\left(\lambda_{1}+\lambda_{4}\right) \mathrm{e}^{-s \lambda_{2}}}{\log _{10} \lambda_{2}-\mathrm{e}^{-s \lambda_{1}} /\left(1+s \cos \lambda_{4}\right)} \\
& +\frac{1-\mathrm{e}^{-s \lambda_{3}}}{s \lambda_{3}\left(1+\left(s \lambda_{3} / 4 \pi\right)^{2}\right)}
\end{aligned}
$$

where the box of fundamental system parameters is given by $\lambda_{1}^{0} \in[9.25,9.35], \lambda_{2}^{0} \in[3,5], \lambda_{3}^{0} \in[0.5,0.7]$, and $\lambda_{4}^{0} \in[0.49,0.5]$. The template of the above uncertain transfer function is required at $\omega=0.5$. For the purpose of computations, the algorithm is implemented in the programming language PASCAL-XSC (Klatte et al., 1993). With the choice $\varepsilon_{x}=0.005$, the algorithm generated 7481 solution (angle-magnitude) rectangles covering the template, in about $10 \mathrm{~min}$ on a PC. For comparison purposes, a finite point approximation to the 'actual' template is also generated using MATLAB and simple gridding, with an 8-point grid for each parameter. For clarity, only the boundary of the template generated using the proposed algorithm is plotted versus the MATLAB template in Fig. 1. The template generated using the algorithm is seen to be quite satisfactory.

\section{Conclusions}

An algorithm has been proposed for generation of frequency response templates for the wide class of magnitude and phase functions that are continuous with respect

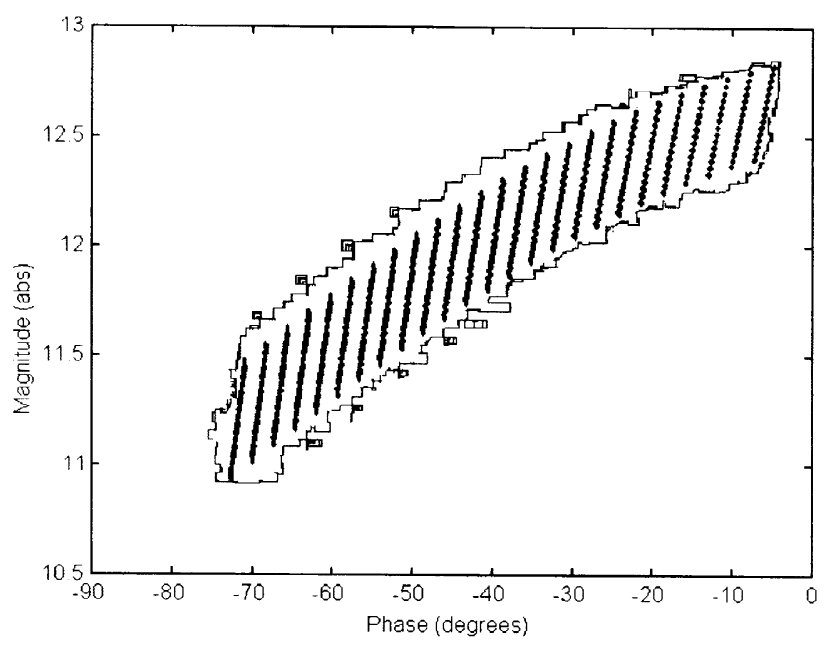

Fig. 1. The boundary of the template generated using the proposed algorithm. The inner points are those generated using MATLAB and simple gridding. to the uncertain system parameters. Other key aspects of the algorithm are that the generated templates are of arbitrary accuracy, safe, and reliable. An illustrative example has been provided.

\section{References}

Ackermann, J., \& Sienel, W. (1991). On the computation of value sets for robust stability analysis. In Proceedings first European control conference, Grenoble, France, 1345-1350.

Bailey, F. N., \& Hui, C. H. (1989). A fast algorithm for computing parametric rational functions. IEEE Transactions on Automatic Control, 34(11), 1209-1212.

Bailey, F. N., Panzer, D., \& Gu, G. D. (1988). Two algorithms for frequency domain design of robust control systems. International Journal of Control, 48(5), 1787-1806.

Bailey, F. N., \& Panzer, D. (1988). A fast algorithm for computing parametric rational functions. In Proceedings of $A C C$, vol. 1 (pp. 22-23), Atlanta, GA, USA.

Barmish, B. R., Ackermann, J., \& Hu, H. (1990). The tree structured decomposition: A new approach to robust stability analysis. In Proceedings of Conference on Information science systems, Princeton, NJ: Princeton University.

Barmish, B. R., \& Tempo, R. (1995). On mappable nonlinearities in robustness analysis. In Proceedings of third. ECC '95, vol. 2, pages Rome, Italy (pp. 1430-1435).

Bartlett, A. C. (1990). Nyquist, Bode, and Nichols plots of uncertain systems. In Proceedings of ACC, volume 2, pages 2033-2036, San Diego, CA, USA.

Bhattacharyya, S. P., Chapellat, H., \& Keel, L. H. (1995). Robust control-The parametric approach. New York: Prentice-Hall.

Cohen, B., Nordin, M., \& Gutman, P. O. (1995). Recursive grid methods to compute value sets for transfer functions with parametric uncertainty. In Proceeedings of ACC (pp. 3861-3865).

Eszter, E. G., \& Pena, R. S. S. (1994). Computation of algebraic combinations of uncertainty value sets. IEEE Transactions on Automatic Control, 39(11), 2315-2318.

Fialho, I. J., Pande, V., \& Nataraj, P. S. V. (1992). Design of feedback systems using Kharitonov's segments in Quantitative Feedback Theory. In Proceedings of First QFT symposium, Dayton, Ohio, USA, (pp. 457-470).

$\mathrm{Fu}$, M. (1990). Computing the frequency response of linear systems with parametric perturbation. Systems and Control Letters, 15(1), $45-52$.

Fu, M., Dasgupta, S., \& Blondel, V. (1995). Robust stability under a class of nonlinear parametric perturbations. IEEE Transactions on Automatic Control, 40(2), 213-223.

Gutman, P. O., Baril, C., \& Neumann, L. (1994). An algorithm for computing value sets of uncertain transfer functions in factored real form. IEEE Transactions on Automatic Control, 39(6), $1268-1273$.

Horowitz, I. M. (1991). Survey of Quantitative Feedback Theory (QFT). International Journal of Control, 53(2), 255-291.

Hwang, C., \& Chen, J. (1995). Computation of the frequency response of interval systems. Circuits, Systems, and Signal Processing, 15(6), 807-818.

Isogai, A., Ohta, Y., \& Haneda, H. (1993). Improvement of polygon interval arithmetic and evaluation of value sets of transfer functions of uncertain systems. Transactions of Institution of Electrical Engineers of Japan, 113-C(10), 772-780.

Kearfott, R. B. (1996). Rigorous global search: Continuous problems. Dodrecht: Kluwer Academic Publishers.

Klatte, R., Kulisch, U., Neaga, M., Ratz, D., \& Ullrich, Ch. (1993). PASCAL-XSC language reference with examples. Berlin: Springer. 
Lan, L. (1994). Construction of the value sets for interval transfer functions and their application to robust control problems. Automation and Remote Control, 55(1) (part 2) 118-129.

Lasky, T. A., \& Ravani, B. (1997). Use of convex hulls for plant template approximation in QFT design. Transactions of the ASME, Journal of Dynamic Systems, Measurement and Control, 119(3), 598-600.

Moore, R. E. (1979). Methods and applications of interval analysis. Philadelphia: SIAM.

Neumaier, A. (1988). The enclosure of solutions of parameter dependent systems of equations. In R. E. Moore, Reliability in computing: The role of interval methods in scientific computations. New York: Academic Press.

Neumaier, A. (1990). Interval methods for systems of equations. Cambridge, England: Cambridge University Press.
Ohta, Y. (1996). Analysis and design of robust control systems via polygon interval arithmetic. Journal of Society of Instrument and Control Engineers, 35(10), 757-762.

Ohta, Y., Gong, L., \& Haneda, H. (1994). Polygon interval arithmetic and interval evaluation of value sets of transfer functions. IEICE Transactions on Fundamentals of Electronics, Communication and Computer Sciences, E77-A (6);1033-1042.

Ratschek, H. (1985). Inclusion functions and global optimization. Mathematical Programming, 3, 300-317.

Sardar, G., \& Nataraj, P. S. V. (1997). A template generation algorithm for non-rational transfer functions in QFT designs. In Proceedings of 36th IEEE Conference on Decision and Control, San Diego, USA (pp. 2684-2689).

Tucker, A. (1980). Applied combinatorics. New York: Wiley. 